RIIT Vol.X. No.4. 2009 363-372, ISSN 2594-0732, FI-UNAM

(artículo arbitrado)

DOI: http://dx.doi.org/10.22201/fi.25940732e.2009.10n4.033

\title{
Efecto de la imidazolina [IM-NH17] en el proceso de corrosión del acero api51-x52 en salmueras acidificadas
}

\section{Effect of an Imidazoline [IM-NH17] on the Corrosion Process of API51-X52 Steel Exposed in Acidified Brines}

\author{
A.G. Reynaud- Morales \\ Centro de Investigación en Materiales Avanzados, \\ Chihuahua, Chihuahua, México. \\ E-mail: adriana.reynaud@gmail.com \\ M. Casales-Díaz \\ Centro de Ciencias Físicas, UNAM. \\ Cuernavaca, Morelos, México. \\ E-mail:Casales-Díaz.mcasales@fis.unam.mx \\ J.G. Chacón-Nava \\ Centro de Investigación en Materiales Avanzados, \\ Chihuahua, Chihuahua, México. \\ E-mail (autorparacorrespondencia):jose.chacon@cimav.edu.mx \\ L. Martínez-Gómez \\ Centro de Ciencias Físicas, UNAM. \\ Cuernavaca, Morelos, México. \\ E-mail:Img@corrosionyproteccion.com \\ A. Martínez-Villafañe \\ Centro de Investigación en Materiales Avanzados, \\ Chihuahua, Chihuahua, México. \\ E-mail:martinez.villafane@cimav.edu.mx \\ J.G. González-Rodríguez \\ Universidad Autónoma del Estado de Morelos, \\ FCQI-CIICAP, Cuernavaca, Morelos, México. \\ E-mail:ggonzalez@uaem.mx
}

(Recibido: octubre de 2007; aceptado: septiembre de 2008)

\section{Resumen}

Se estudia la eficiencia de inhibición del compuesto Imidazolina Aminoetil Oleica [IM-NH17]) en la corrosión de un acero API5L-X52 en tres soluciones de salmuera acidificada a $\mathrm{pH} 3$ a una temperatura de $50^{\circ} \mathrm{C}$, empleando pruebas de polarización potenciodinámica y mediciones de resistencia de polarización. Bajo las condiciones de prueba, los resultados de curvas de polarización indicaron que el inhibidor se comporta como un inhibidor de tipo mixto. Los resultados de resistencia de polarización lineal mostraron un incremento en los valores de Rp para las soluciones con 
DOI: http://dx.doi.org/10.22201/fi.25940732e.2009.10n4.033

Efecto de la imidazolina [IM-NH17] en el proceso de corrosión del acero api5I-x52 en salmueras acidificadas

inhibidor en comparación con el valor registrado para la solución de referencia sin inhibidor. Así, se encontró que la eficiencia del inhibidor aumenta en el rango de 12.5 ppm a 25 ppm. También, de los resultados se deriva que el modelo de isoterma de Langmuir es el mecanismo de adsorción que mejor se ajusta al presente sistema.

Descriptores: Acero X-52, imidazolinas, resistencia de polarización, polarización potenciodinámica,isoterma de adsorción.

\begin{abstract}
The inhibition effect of an aminoethyl oleic imidazoline [IM-NH17]) on the corrosion of API5L-X 52 steel in acidified $5 \% \mathrm{NaCl} \mathrm{pH} 3$ solutions at $50^{\circ} \mathrm{C}$ was studied via electrochemical methods, i.e. potentiodynamic polarization tests and linear polarization resistance measurements. Polarization curves revealed that, under the test conditions, the inhibitor performs as a mixed type inhibitor. Linear Polarization Resistance measurements showed an increase in $R_{p}$ values for inhibitor containing solutions as compared with the blank solution. The results revealed that the inhibitor efficiency increases with inhibitor concentration in the range from $12.5 \mathrm{ppm}$ to $25 \mathrm{ppm}$. From the electrochemical results, it was found that the adsorption mechanism that better fits the present system follows the Langmuir isotherm model.
\end{abstract}

Keywords: X-52 steel, imidazoline, linear polarization resistance, potentiodynamic polarization, adsorption isotherm.

\section{Introducción}

Durante las dos últimas décadas se ha tenido un aumento considerable en la investigación y desarrollo de nuevos inhibidores de corrosión de baja toxicidad (Hackerman y Snavely, 1984, Bentiss et al., 1999 y 2004). Esfuerzos en todo el mundo han sido encaminados a la búsqueda de compuestos más adecuados que cumplan con las restricciones impuestas a sustancias peligrosas. En general, los inhibidores de corrosión encuentran un amplio campo de aplicación prácticamente en todo tipo de industrias. En particular, compuestos base imidazolinas tienen amplia aplicación en la industria petrolera en operaciones de producción, transporte y refinación de hidrocarburos (Bondareva et al., 2004), así como también en la industria de procesos químicos donde formulaciones ácidas ( $\mathrm{HCl}$, ácidos orgánicos etc.) son ampliamente usadas para remover encostramiento por acumulación de óxidos o sales en los equipos de proceso (Baril et al., 2000, Edwards et al., 1994, Kreuser et al., 1997).

Hoy en día, compuestos orgánicos base nitrógeno se cuentan entre los de mayor uso como inhibidores de corrosión para algunas de las aplicaciones mencionadas. Algunas opciones para tener menores niveles de toxicidad implican el uso de compuestos tales como los alifáticos formadores de película, las aminas aromáticas así como las imidazolinas (Gough et al., 2002). Así, a fin de tener un mejor entendimiento de las características de baja toxicidad y comportamiento de inhibidores a un costo aceptable, se requiere una mejor comprensión del mecanismo de inhibición. Es generalmente aceptado que la inhibición de compuestos tales como imodazolinas resulta de la adsorción de películas del inhibidor sobre la superficie metálica (Cruz et al., 2004). La adsorción de esas moléculas sobre la superficie del acero al carbono ocurre por transferencia de carga parcial entre la molécula y el metal, formando un enlace covalente. En moléculas N-heterocíclicas, esta transferencia de carga es promovida por el par de electrones solitarios presentes en los átomos de hidrógeno de la molécula orgánica (Edwards et al., 1994, Morales et al., 2004). Otra forma de adsorción tiene lugar vía atracción electrostática entre un átomo de hidrógeno cargado positivamente y la superficie metálica cargada negativamente. Diversos autores (Bahadur, 1993, Edwards et al., 1994, Morales et al., 2004) han estudiado diferentes aspectos estructurales de imidazolinas y sus mecanismos de adsorción e inhibición, p. ej. a) el efecto de la longitud de la cadena hidrofóbica (cola), b) la química del grupo principal (anillo imidazolina), y c) la hidrólisis de la imidazolina.

No obstante, el importante trabajo reportado en la literatura sobre el efecto inhibidor de imidazolinas en la corrosión de los aceros al carbono, requiere de más información en algunos aspectos. Luego entonces, el objetivo del presente trabajo es investigar las propiedades de 
DOI: http://dx.doi.org/10.22201/fi.25940732e.2009.10n4.033

A.G. Reynaud- Morales, M. Casales-Díaz, J.G. Chacón-Nava, L. Martínez-Gómez, A. Martínez-Villafañe y J.G. González-Rodríguez

inhibición de una imidazolina de tipo oleico empleada usualmente en ambientes ácidos, así como su probable mecanismo de protección mediante el uso de parámetros termodinámicos y datos de cinética de adsorción. La información generada es importante porque contribuye al conocimiento sobre el tipo particular de protección al metal y su eficiencia frente a la corrosión para un tipo especifico de acero al carbono muy empleado en la industria petroquímica.

\section{Procedimiento experimental}

Se prepararon muestras rectangulares $(1 \mathrm{~cm} \times 1 \mathrm{~cm} \times$ $0.5 \mathrm{~cm}$ ) de un acero API5L-X52 para ser empleadas como electrodos de trabajo en las mediciones electroquímicas. La composición del acero empleado se presenta en la tabla 1.

Las muestras fueron montadas en resina epóxica y desbastadas con lija de $\mathrm{SiC}$ desde grado 300 hasta grado 1200, lavando la superficie de trabajo con agua bidestilada, desengrasando con acetona y secando con una corriente de aire caliente antes de efectuar las pruebas. El electrolito empleado consistió en una solución de $\mathrm{NaCl}$ (grado analítico) al $5 \%$ acidificada a un $\mathrm{pH}=3$ con una solución de $\mathrm{HCl}(\sim 0.2 \mathrm{M}$, preparada con reactivo grado analítico). Esta solución fue usada como blanco para propósitos de comparación de resultados. Para preparar las soluciones conteniendo inhibidor, se empleó agua desmineralizada. En este estudio una imidazolina aminoetil oleica [IM-NH17]) Lakeland Labs., se empleó como inhibidor de corrosión.

Se emplearon dos concentraciones de inhibidor: 12.5 ppm y $25 \mathrm{ppm}$. En cada experimento se hizo uso de solución fresca. Una celda estándar de vidrio $(0.5 \mathrm{Lt})$ de cuatro bocas fue empleada en los experimentos. Las mediciones electroquímicas se realizaron empleando un potenciostato/galvanostato ACM Instruments acoplado a una PC con el software asociado para adquisición y análisis de datos. Un arreglo convencional de tres electrodos fue empleado: muestras de acero API5L-X52 (con área expuesta de aproximadamente $1 \mathrm{~cm}^{2}$ ) como electrodo de trabajo (ET); un electrodo de calomel saturado como electrodo de referencia (SCE) y una barra de grafito como contraelectrodo (CE). Las mediciones, empleando la técnica de resistencia de polarización lineal (RPL), se llevaron a cabo aplicando un pequeño barrido de potencial de $-10 \mathrm{mV} \mathrm{a}+10 \mathrm{mV}$ alrededor del potencial de corrosión ( $\mathrm{E}_{\text {corr }}$ ) a una velocidad de barrido de 1.5 $\mathrm{mVs}^{-1}$. Se obtuvieron curvas de polarización en un rango de $-1000 \mathrm{mV}$ a $+350 \mathrm{mV}$ a una velocidad de barrido de $1.5 \mathrm{mVs}^{-1}$.

En todos los casos, las mediciones se efectuaron a una temperatura de $50{ }^{\circ} \mathrm{C}(323.16 \mathrm{~K})$, la cual fue controlada usando una mantilla de calentamiento digital y un termómetro.

A fin de determinar el mecanismo de adsorción del inhibidor empleado (IM-NH17) sobre el acero API5L-X52, se efectuó un ajuste mediante regresión lineal empleando valores de cobertura superficial $(\theta)$. Este valor fue estimado de los resultados de densidad de corriente obtenidos de las pruebas potenciodinámicas, asumiendo un proceso de corrosión uniforme en el electrodo de trabajo. Una vez que se tiene la isoterma más adecuada, la constante de adsorción, $K_{\text {ad }}$ puede ser estimada, y por tanto, también la energía libre de adsorción de Gibbs $\left(\Delta G_{a d}^{\circ}\right)$ dada por:

$$
\Delta G_{a d}^{\circ}=R T \cdot \operatorname{In}\left(K_{a d}\right)
$$

donde $R$ es la constante ideal de los gases $\left(8.314 \times 10^{-3}\right.$ $\mathrm{kJmol}^{-1} \cdot \mathrm{K}^{-1}$ ) y $\mathrm{T}$ es la temperatura absoluta en ${ }^{\circ} \mathrm{K}$.

\section{Resultados y discusión}

$$
\text { Curvas de polarización }
$$

La figura 1 muestra las curvas de polarización obtenidas durante un tiempo de inmersión de 4 hr. Aquí, se observa que la adición de inhibidor (IM-NH17) produce los siguientes efectos: primero, la adición y posterior incremento de la concentración de inhibidor produce un cambio considerable hacia valores menores de densidad de corriente; segundo, los potenciales de corrosión de las soluciones con inhibidor cambian hacia valores más nobles, siendo esto indicativo del carácter anódico del inhibidor.

Tabla 1. Composición química del acero API5L-X52 (\% peso)

\begin{tabular}{ccccccc}
\hline $\mathrm{C}$ & $\mathrm{P}$ & $\mathrm{Mn}$ & $\mathrm{S}$ & $\mathrm{V}$ & $\mathrm{Ti}$ & $\mathrm{Fe}$ \\
\hline 0.31 & 0.04 & 1.35 & 0.05 & 0.02 & 0.03 & $\mathrm{Ba}$ \\
\hline
\end{tabular}




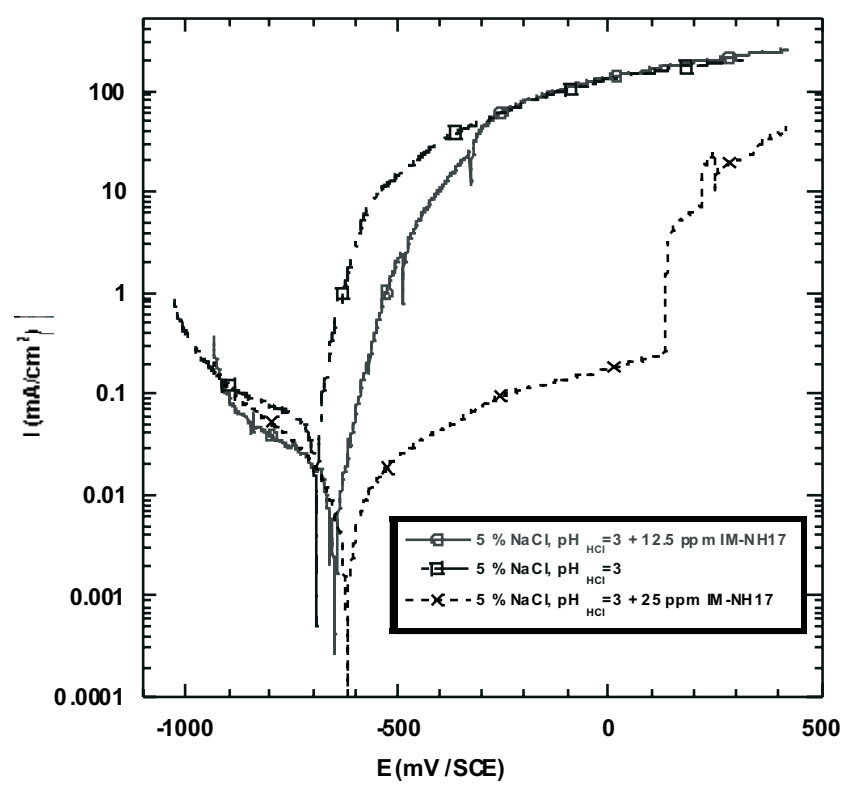

Figura 1. Curvas de polarización obtenidas para el acero API5L-X52 expuesto en las diferentes soluciones con y sin inhibidor

La eficiencia de inhibición (\%E.I) para cada concentración de inhibidor fue determinada de acuerdo a la ecuación:

$$
\% E I=\frac{i_{\text {corr }}^{0}-i_{\text {corr }}^{\text {inh }}}{i_{\text {corr }}} \times 100
$$

donde $i_{\text {corr }}{ }^{0}$ e $i_{\text {corr }}{ }^{\text {inh }}$ son los valores de densidad de corriente en ausencia y con inhibidor, respectivamente.

Los parámetros electroquímicos obtenidos de las curvas de polarización se tienen en la tabla 2. Para la concentración de $12.5 \mathrm{ppm}$ la curva anódica exhibe un valor de pendiente anódica $\beta_{\mathrm{A}}$ similar al obtenido por la solución de referencia (sin inhibidor) a bajos sobrepotenciales (de $\mathrm{E}_{\text {corr }}$ a $\sim-500 \mathrm{mV}$ ). A mayores sobrepotenciales (de $\sim-500 \mathrm{a} \sim-300 \mathrm{mV}$ ) el valor de la pendiente es aún mayor, pero menor que la pendiente correspondiente a la solución de referencia. A sobrepotenciales del orden de -250 mV y por arriba, la curva anódica se une a la correspondiente a la de la solución blanco. Aquí, este efecto podría ser atribuido a una desorción de las moléculas de IM-NH17 de la superficie metálica probablemente debido a interacciones de carga de las moléculas adsorbidas con otras moléculas de inhibidor de la solución en volumen. Estas interacciones desvían el sistema sustrato-adsorbato de las condiciones de equilibrio y serán promovidas por las altas densidades de corriente positiva producidas en sitios anódicos, ya que una parte del numero total de sitios anódicos no es cubierta por el inhibidor o productos de corrosión (liberación de iones $\left.\mathrm{Fe}^{+2} \circ \mathrm{Fe}^{+3}\right)$, luego entonces, el valor de $(\theta)$ no será un máximo (Frenier, 1998, Jovancicevic et al., 1999, Baril et al., 2000). Por otra parte, a una concentración de inhibidor de 25 ppm, se observó la menor densidad de corriente anódica y el valor de la pendiente $\beta_{\mathrm{A}}$ fue el más alto en las regiones de sobrevoltaje bajo y medio (desde Ecorr a -300 mV), con una pendiente prácticamente igual a la de la solución blanco a valores de potencial más positivos que $-250 \mathrm{mV}$. A esta concentración de inhibidor, valores bajos de densidad de corriente se observaron en todo el barrido anódico. Este efecto hace pensar que, en este caso, el equilibrio de adsorción se mantiene, debido a la formación de una película de IM-NH17 y alguna cantidad de productos de corrosión. Esta película debe mantener el equilibrio de adsorción del sistema de ser perturbado por cualquier interacción de carga, reduciendo la magnitud de corriente positiva. A $25 \mathrm{ppm}$ de inhibidor, la máxima cobertura superficial $(\theta)$ se alcanza, dado el importante papel que tiene la formación de productos de corrosión a esta concentración. Este hecho es soportado por la observación de que a 25 ppm de inhibidor productos de corrosión con características protectoras se forman con mayor rapidez (como lo denotan los valores mas altos de $\beta_{A}$ ) al comienzo de la polarización anódica. Entonces, para este tipo de solución, la concentración óptima de inhibidor debe estar 
DOI: http://dx.doi.org/10.22201/fi.25940732e.2009.10n4.033

A.G. Reynaud- Morales, M. Casales-Díaz, J.G. Chacón-Nava, L. Martínez-Gómez, A. Martínez-Villafañe y J.G. González-Rodríguez

alrededor de 25 ppm (Luo et al., 1998, Jovancicevic et al., 1999). A esta concentración de IM-NH17, las curvas de polarización sugieren que la eficiencia de inhibidor observada puede ser relacionada con la marcada inhibición del proceso anódico (disolución del metal) y el proceso catódico (evolución de hidrógeno). Los resultados del presente trabajo corresponden bien con el estudio de Szyprowski (2000), donde se reporta que para acero al carbono 1020 y acero inoxidable 304 en una solución de $\mathrm{NaCl}$ al $2 \%$ y varios tipos de imidazolinas a $40^{\circ} \mathrm{C}$, los inhibidores mostraron la mayor eficiencia de protección a una concentración de $25 \mathrm{ppm}$.

La figura 2 muestra un esquema del mecanismo de absorción del IM-NH17 en el dominio anódico. Este mecanismo puede darse como sigue: la molécula de inhibidor se fija en los iones $\mathrm{Fe}^{+3} \mathrm{O} \mathrm{Fe}^{+2}$ de la red cristalina del óxido de la superficie metálica por medio de los átomos de nitrógeno del anillo de imidazolina sin atar ninguna cadena exterior N3, como ha sido propuesto por (Edwards et al., 1994, Ramachandran et al., 1997, Ramachandran et al., 1999). Luego, el átomo N3 comparte su par de electrones con los iones férrico o ferroso de la superficie porosa del óxido.
La tendencia mostrada por las curvas catódicas indica que el efecto de inhibición no fue tan marcado como en la región anódica y sólo pequeñas diferencias fueron notadas en las soluciones conteniendo inhibidor. Sin embargo, un cambio en la pendiente catódica, $\beta_{\mathrm{c}}$, se observó en comparación con la solución blanco: la reacción de descarga protónica parece disminuir con la adición de inhibidor a la solución. El valor más bajo de $\beta_{\mathrm{c}}$ fue obtenido a una concentración de 25 ppm (tabla 2). Para explicar esto, la siguiente hipótesis es propuesta: en los sitios catódicos la molécula de inhibidor se hace catiónica mediante el enlace de un ion $\mathrm{H}^{+}$donando el par de electrones no compartidos del átomo de nitrógeno en el grupo funcional de la cadena amino-etílica (N8). Esta reacción de protonación se muestra en la figura 3.

Una vez que esta reacción tiene lugar, la molécula IM-NH17- $\mathrm{H}^{+}$es unida a la superficie del metal cargada negativamente, formando una película protectora, bloqueando sitios anódicos activos y limitando el transporte de iones $\mathrm{H}^{+}$o moléculas de $\mathrm{O}_{2}$ en la superficie metálica. En soluciones que contienen altas concentraciones de iones $\mathrm{Cl}^{-}$, como en el presente estudio, es razonable asumir que la superficie cargada negativamente consiste de iones $\mathrm{Cl}^{-}$adsorbidos.

Tabla 2. Parámetros electroquímicos y E.I. \% obtenidas de las mediciones de polarización potenciodinámica para el acero API5L-X52 expuesto a las diferentes soluciones base $\mathrm{NaCl} 5 \%$

\begin{tabular}{cccccc}
\hline Solución & $\begin{array}{c}E_{\text {corr }} \\
(\mathrm{mV} \text { vs. SCE })\end{array}$ & $\begin{array}{c}i_{\text {corr }} \\
\left(\mathrm{mA} / \mathrm{cm}^{2}\right)\end{array}$ & $\begin{array}{c}\beta_{a} \\
(\mathrm{mV} / \mathrm{dec})\end{array}$ & $\begin{array}{c}\beta_{c} \\
(\mathrm{mV} / \mathrm{dec})\end{array}$ & $\begin{array}{c}\text { E.I. } \\
(\%)\end{array}$ \\
\hline $\begin{array}{c}5 \% \mathrm{NaCl}, \\
(\mathrm{Blanco})\end{array}$ & -700 & 0.060 & 100 & 450 & -- \\
$\begin{array}{c}5 \% \mathrm{NaCl}, \\
+12.5 \mathrm{ppm}\end{array}$ & -650 & 0.015 & 85 & 400 & 75.0 \\
$\mathrm{IM}-\mathrm{NH} 17$ & & & & & \\
$\begin{array}{r}5 \% \mathrm{NaCl}, \\
+25 \mathrm{ppm}\end{array}$ & -620 & 0.010 & 400 & 240 & 83.3 \\
IM-Nh17 & & & & & \\
\hline
\end{tabular}

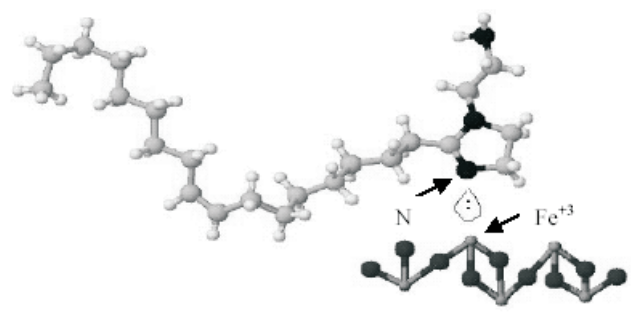

Figura 2. Molécula de IMNH-17 unida a la superficie metálica por medio de un enlace Fe-N 


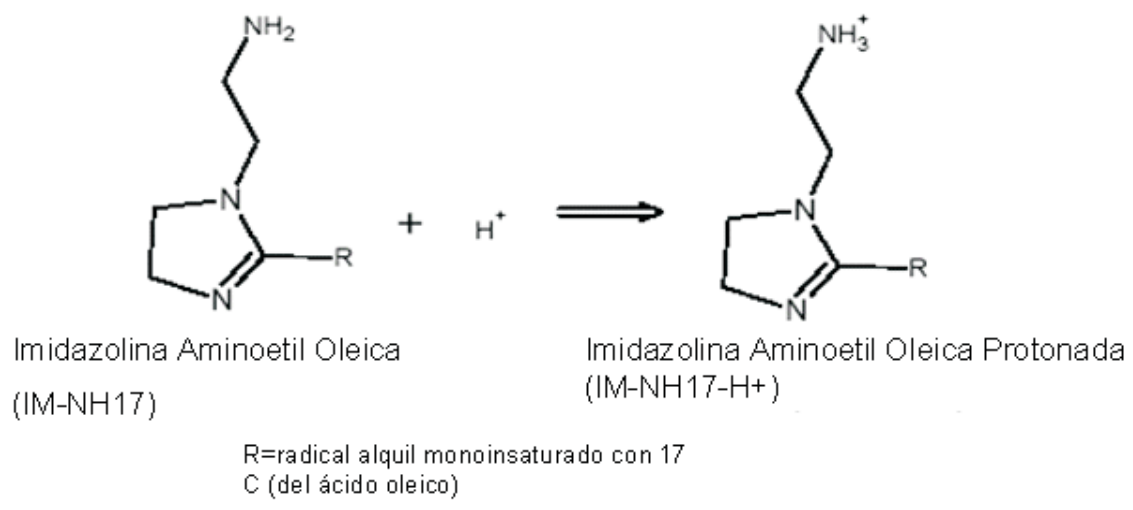

Figura 3. Reacción de protonación del inhibidor IM-NH17

\section{Resistencia de polarización lineal}

Esta técnica representa un medio conveniente para estimar velocidades de corrosión cuando las pendientes de Tafel $\beta_{a}$ y $\beta_{c}$ son medidas o estimadas de resultados de la literatura para materiales en condiciones similares de exposición a un medio ambiente específico. Una ventaja importante de esta técnica es el evitar perturbación del sistema (polarización) en un amplio rango de potenciales. Así, dentro de un rango de $\pm 15 \mathrm{mV}$ del potencial libre de corrosión, se conoce que la densidad de corriente aplicada es una función lineal del potencial de electrodo (Stern and Geary, 1957). Entonces, la pendiente de $(d E / d i)$ se conoce como la resistencia de polarización, $R p$, relacionándose con los parámetros del sistema en la forma:

$$
\frac{d E}{d i}=R_{p}=\frac{\beta_{a c}}{2.3\left(\beta_{a}+\beta_{c}\right) i_{\text {corr }}}
$$

donde $\beta_{a}$ y $\beta_{c}$ son las pendientes de Tafel anódica y catódica $\left(\right.$ en $\mathrm{mVdec}^{-1}$ ) respectivamente, $i_{\text {corr }}$ es la densidad de corriente de corrosión en $\mathrm{mAcm}^{-2}$ y $R p$ es la resistencia de polarización en $\Omega \cdot \mathrm{cm}^{2}$.

Esta es la ecuación de Stern-Geary, ampliamente usada para estimar el valor de $i_{\text {corr }}$. Dado que no es posible obtener las pendientes de Tafel por este método, en el presente trabajo estos valores fueron derivados de las curvas de polarización potenciodinámicas, ver tabla 2 . Luego entonces, una vez determinando el valor de Rp, es posible calcular el valor de $i_{\text {corr }}$. En general, los valores estimados $i_{\text {corr }}$, así como los valores de eficiencia de inhibidor (\%E.I.) están de acuerdo con los valores calculados de las curvas de polarización (tabla 2). Para la solución con 12.5 ppm de IM-NH-17, los datos de la tabla 3 indican que el valor de $\mathrm{Rp}$ es casi 4 veces mayor que el medido para la solución blanco y casi 3 veces menor que para la solución con 25 ppm de inhibidor. A esta última concentración, el alto valor de $R_{p}$ y el bajo valor de $\beta_{c}$ contribuyen a tener un valor alto de la pendiente anódica $\beta_{a}$, con un efecto general de un decremento en la velocidad de corrosión $\left(i_{\text {corr }}\right)$. De manera general, podemos decir que los datos obtenidos de RPL y curvas de polarización son consistentes entre sí (tablas 2 y 3 ). Los valores de eficiencia calculados para el inhibidor aquí empleado coinciden bien con los resultados para imidazolinas sobre acero al carbono 1018, presentados por Zhao et al. (2004).

\section{Mecanismo de adsorción}

Por mucho tiempo se ha pensado que los mecanismos de inhibición de corrosión por moléculas orgánicas (p.ej. aminas, imidazolinas, mercaptanos, etc) ocurren vía el fenómeno de adsorción (Hackerman et al., 1984, Zvaya et al., 1994, Ramachandran et al., 1997). Así, diferentes modelos de isotermas de adsorción tales como la isoterma de Frumkin, la isoterma de Langmuir y la isoterma de Temkin han sido propuestos (Zvaya et al., 1994). Las ecuaciones correspondientes para cada modelo son:

$$
\begin{array}{ll}
\text { Isoterma de Temkin: } & K_{a d} C=e^{f \theta} \\
\text { Isoterma de Langmuir: } & K_{a d} C=\frac{\theta}{1-\theta} \\
\text { Isoterma de Frumkin: } & K_{a d} C=\left(\frac{\theta}{1-\theta}\right) e^{f \theta}
\end{array}
$$

Donde $K_{a d}$ es la constante de equilibrio de adsorción, $C$ es la concentración de inhibidor, $f$ es la constante de 
interacción molecular y $\theta$ es la cobertura superficial. A fin de ligar un ion $\mathrm{Fe}^{+2} \mathrm{O} \mathrm{Fe} e^{+3}$, cualquier sustancia polar debe desplazar moléculas de agua de la superficie metálica (Kreuser et al., 1997, Morales et al., 2004). Este es el caso de la imidazolina, dado por la siguiente reacción que describe el equilibrio de adsorción para el inhibidor IMNH-17 en la forma.

$$
I M-N H 17_{(a q)}+n H_{2} \mathrm{O}_{(a d s)} \Longleftrightarrow I M-N H 17_{(a d s)}+n H_{2} O_{(a q)}
$$

Ahora bien, para determinar la isoterma de adsorción que mejor se ajuste a nuestro sistema, debemos asumir que un proceso de corrosión uniforme ocurre en el electrodo de trabajo. Entonces, la velocidad de corrosión para las muestras en solución con inhibidor deberá ser igual al número de especies activas que permanecen sin obstruir o sin bloquear después de que ocurre la adsorción de moléculas del inhibidor. La cobertura superficial, $\theta$, puede entonces ser calculada por la ecuación (Atkins, 1980, Altura y Nobe, 2004):

$$
\theta=\frac{i_{\text {corr }}{ }^{0}-i_{\text {corr }}{ }^{i n h}}{i_{\text {corr }}{ }^{0}}
$$

donde $\mathrm{i}_{\text {corr }}{ }^{0}$ y $\mathrm{i}_{\text {corr }}{ }_{\text {inh }}$ son los valores de densidad de corriente en ausencia $y$ en presencia de inhibidor, respectivamente.

Una vez calculados los valores de $\theta$ a cada concentración de inhibidor, se efectuó un análisis de regresión lineal para esos datos. De la figura 4 se observa fácilmente que el modelo de Langmuir (ecuación 5) parece ser el más adecuado a nuestros datos experimentales. A fin de confirmar esto, se graficó la forma linearizada de la ecuación 6 usando datos de varias corridas experimentales incluyendo una nueva concentración más alta de inhibidor, es decir, $37.5 \mathrm{ppm}$.

Esta concentración de inhibidor dio como resultado un valor de $\theta$ de $\sim 0.8416$, es decir, ligeramente por arriba del obtenido para 25 ppm ( 0.833). De los resultados de la figura 5 podemos decir que el caso más general (isoterma de Frumkin) puede ser rechazado, dado que el valor de $f$ se aproxima a cero (Baril et al., 2000). Entonces, los datos pueden ajustarse bien a la isoterma de adsorción de Langmuir, obteniendo un coeficiente de correlación de $\mathrm{R}^{2}=0.986$. Herrag (2007) en su trabajo con derivados de alquil aminas en solución ácida también ha reportado que la isoterma de adsorción que mejor se ajusta a sus datos experimentales de pérdida de peso es el modelo de isoterma de Lagmuir.

Para nuestro sistema, (acero API5L-X52-IM-NH17), la figura 4 muestra el valor derivado de la constante de adsorción $\left(K_{a d}\right)$. Este valor es la pendiente inversa en la ecuación de la línea recta correspondiente al ajuste de los datos experimentales (obtenidos de $\mathrm{i}_{\text {corr }}{ }^{0}$ y $\mathrm{i}_{\text {corr }}$ inh con el modelo de Langmuir (ecuación 4). De los valores $K_{a d}$ Y la ecuación 1, se calculó un valor de energía libre de adsorción $\Delta G_{a d}^{o} \approx-30 \mathrm{kJmol}^{-1} \mathrm{y}$ este valor nos indica un proceso de adsorción espontánea del inhibidor sobre la superficie metálica. El valor calculado para $\Delta G^{\circ}{ }_{a d}$ corresponde bien con el valor reportado recientemente por Durnie et al. (2005).

Tabla 3. Parámetros electroquímicos y E.I.\% obtenidas de las mediciones de resistencia de polarización lineal para el acero APISL-X52 expuesto a las diferentes soluciones base $\mathrm{NaCl} 5 \%$

\begin{tabular}{ccccc}
\hline Solución & $\begin{array}{c}E_{\text {corr }} \\
(\mathrm{mV} \text { vs. SCE })\end{array}$ & $\begin{array}{c}R_{p} \\
\left(\Omega \mathrm{cm}^{2}\right)\end{array}$ & $\begin{array}{c}\mathrm{i}_{\text {corr }} \\
\left(\mathrm{mA} / \mathrm{cm}^{2}\right)\end{array}$ & $\begin{array}{c}\text { E.I. } \\
(\%)\end{array}$ \\
\hline $\begin{array}{r}5 \% \mathrm{NaCl}, \\
(\mathrm{Blanco})\end{array}$ & -702 & 565 & 0.0629 & - \\
$\begin{array}{r}5 \% \mathrm{NaCl}, \\
+12.5 \mathrm{ppm}\end{array}$ & -653 & 2146 & 0.0142 & 77.4 \\
$\mathrm{IM}-\mathrm{NH17}$ & & & & \\
$5 \% \mathrm{NaCl}$, & & & & \\
$+25 \mathrm{ppm}$ & -625 & 6097 & 0.0107 & 82.9 \\
IM-Nh17 & & & & \\
\hline
\end{tabular}




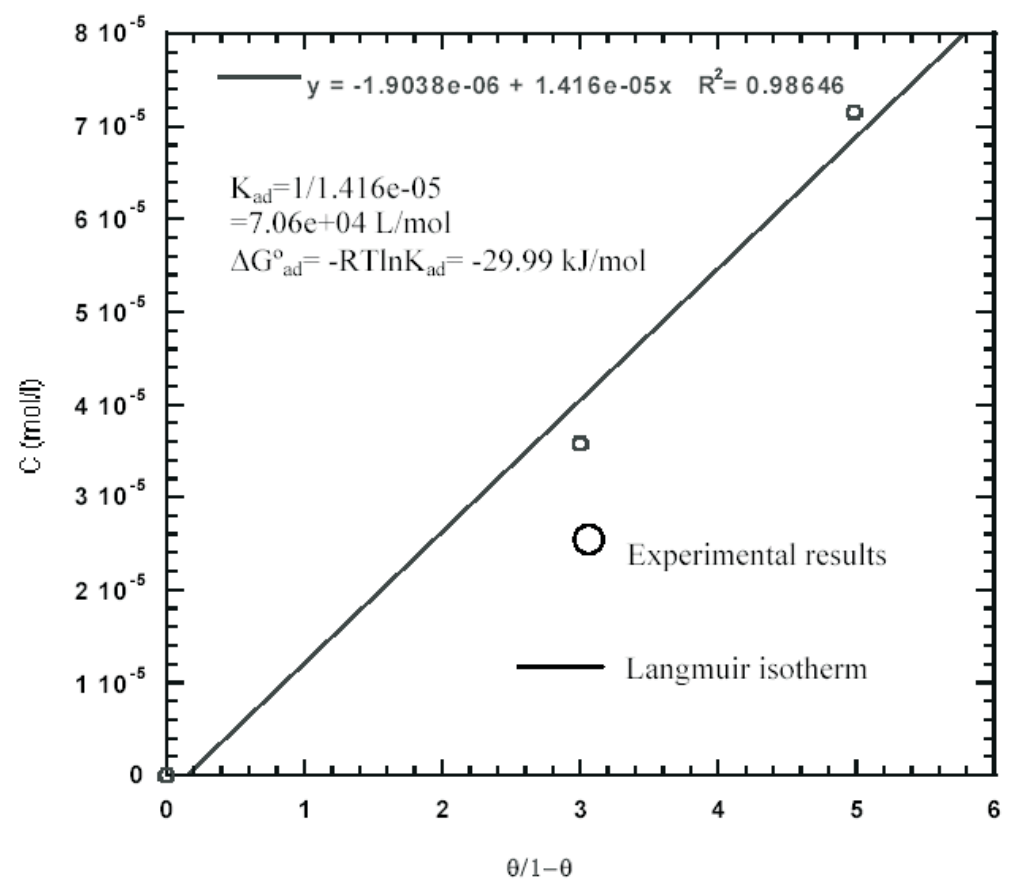

Figura 4. Resultados experimentales de la isoterma de adsorción para el inhibidor IM-NH17 a dos concentraciones $\left(12.5 \mathrm{ppm}=3.58 \times 10^{-5} \mathrm{M}\right.$, y $\left.25 \mathrm{ppm}=7.16 \times 10^{-5} \mathrm{M}\right)$ en un acero API5L-X52

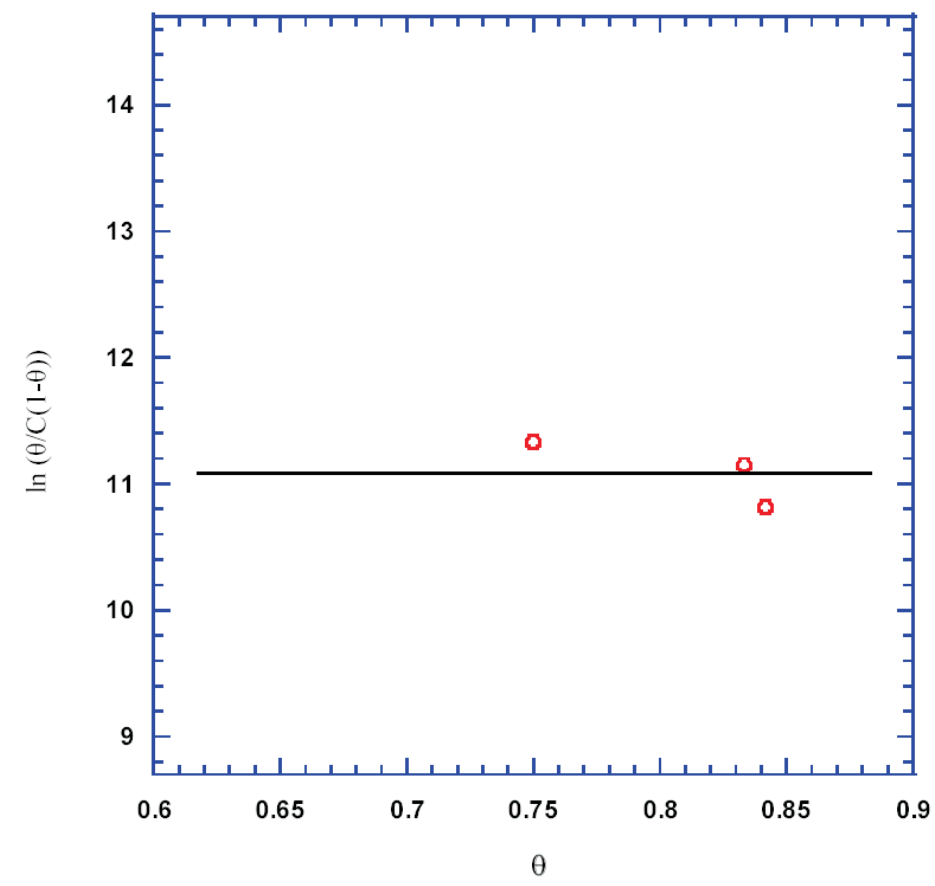

Figura 5. Gráfico de resultados experimentales de la isoterma de adsorción para el inhibidor IM-NH17 a tres concentraciones (12.5 ppm, $25 \mathrm{ppm}$ y $37.5 \mathrm{ppm}$ ) en una solución de $\mathrm{NaCl}$ (5\%) a pH 3 
DOI: http://dx.doi.org/10.22201/fi.25940732e.2009.10n4.033

A.G. Reynaud- Morales, M. Casales-Díaz, J.G. Chacón-Nava, L. Martínez-Gómez, A. Martínez-Villafañe y J.G. González-Rodríguez

\section{Conclusiones}

El compuesto IM-NH17 es una imidazolina aminoetil oleica que ofrece buena protección al acero API5L-X52 en soluciones acuosas de $\mathrm{NaCl}(5 \%)$ acidificadas. A una concentración de $25 \mathrm{ppm}$, la eficiencia de inhibición es del $83 \%$ bajo las condiciones del presente trabajo.

En el dominio anódico la molécula de IM-NH17 se adsorbe a la superficie metálica mediante interacciones donor-aceptor entre un átomo de nitrógeno del anillo imidazolina y un ion férrico o ferroso del óxido formado en la superficie del acero. En el dominio catódico el efecto catiónico del compuesto IM-NH17 sugiere que la molécula se protona mediante el amarre de un ion hidrogeno de la solución. Este proceso parece ocurrir mediante la donación del par de electrones del átomo de nitrógeno en el grupo funcional aminoetil de la cadena. Después de protonarse, la adsorción del IM-NH17 será dada por interacción electrostática con la superficie metálica negativamente cargada.

El inhibidor IM-NH17 actúa bloqueando sitios activos en el metal (inhibición de tipo mixto), pero también reduciendo la cinética de descarga protónica (evolución de hidrogeno) y suprimiendo el proceso de disolución (reacción anódica).

La técnica de resistencia de polarización lineal mostró un aumento en los valores de $\mathrm{R}_{\mathrm{p}}$ con la adición e incremento de la concentración de inhibidor. En general, los resultados derivados con esta técnica y los obtenidos de las curvas de polarización fueron concordantes entre si.

El inhibidor de corrosión IM-NH17 se comporta de acuerdo al modelo de Langmuir, con una energía libre de adsorción de $-30 \mathrm{~kJ} / \mathrm{mol}$.

\section{Agradecimientos}

Los autores agradecen al CONACYT-México por el financiamiento para llevar a cabo este trabajo, así como la asistencia técnica de J. Lugo-Cuevas y G. Vázquez-Olvera (Cimav).

\section{Referencias}

Altura D., Nobe K. Corrosion, 29:433. 1973.

Atkins P.W. Chemisorbed and Physisorbed Species. Oxford. A Textbook in Physical Chemistry University Press. 1980. Pp. 936-938.

Bahadur A. Corrosion Reviews, 11:106. 1993.

Baril G., Moran F., Pebere N. 9th European Symposium on Corrosion Inhibitors. Ferrara, Italy, Sept., Vol. 2, pp.849. 2000.
Bentiss F., Lagrenee M., Traisnel M., Hornez J.C. Corrosion, 55:968. 1999.

Bentiss F., Traisnel M., Vezin H., Hildebrand H.F., Lagrenee M. Corrosion Sci., 46:2781. 2004.

Bondareva S.O., Lisitskii V.V., Yakovtseva N.I., Murinov Yu. I. Russian Chemical Bulletin, 53:803. 2004

Cruz J., Martínez R., Genesca L., García-Ochoa E. Electroanal. Chem., 566:111. 2004.

Durnie W., De Marco R., Kinsella B., Jefferson A., Pejcic A. J. Electrochem Soc., 152:B1. 2005.

Edwards A., Osborne C., Webster S., Klenerman D., Joseph M., Ostovar P., Doyle M. Corrosion Sci., 36:315. 1994.

Frenier W.W. Corrosion 98. NACE San Diego, Cal. Paper 214. 1998.

Gough M.A., Durnie W.H., Auty E.K., Hedges B. Corrosion 2002. NACE Denver, Co., Paper 02301. 2002.

Hackerman N., Snavely E.S. Corrosion Basics: An Introduction, National Association of Corrosion Engineers, Chapter Inhibitors. 1984.

Herrag L. Hammouti B., Aouniti A., El Kadiri S., Touzani R. Acta Chim Slov., 54:419. 2007.

Jovancicevic V., Ramachandran S., Prince P. Corrosion, 55:449. 1999.

Kreuser R.T.,Vanlaer A., Damour A. Corrosion 97. NACE, New Orleans, LA., Paper 409. 1997.

Luo H., Guan Y.C., Han K.N. Corrosion, 54:721. 1998.

Morales-Gil P., Negrón-Silva G., Romero-Romo M., Ángeles-Chávez C., Palomar-Pardavé M. Electrochim. Acta., 49:4733. 2004.

Ramachandran S., Jovancicevic V. Corrosion, 55:259. 1999.

Ramachandran S., Tsai B.L., Blanco M., Chen H., Tang Y., Goddard III W.A.J. Phys.Chem. A, 101:83. 1997.

Ramachandran S., Tsai B.L., Blanco M., Chen H., Tang Y., Goddard III W.A. Langmuir, 12:6419. 1996.

Stern M., Geary A.L. Journal of Electrochemical Society, 104:56. 1957.

Svauya R., Dawson J.L. J. Appl. Electrochem., 24:943. 1994.

Szyprowski A.J. Brit. Corr. J., 35:155. 2000.

Zhao L., Teng H.K., Yang Y.S., Tan X. Materials and Corrosion, 55:684. 2004 
DOI: http://dx.doi.org/10.22201/fi.25940732e.2009.10n4.033

Efecto de la imidazolina [IM-NH17] en el proceso de corrosión del acero api5I-x52 en salmueras acidificadas

\section{Semblanza de los autores}

Adriana G. Reynaud-Morales. Obtuvo el grado de doctora en ciencia de materiales por el Centro de Investigación en Materiales Avanzados S.C. Cursó la licenciatura en ingeniería química por la Universidad Veracruzana y tiene una maestría en cinética química aplicada a hidrocarburos por la Université Pierre et Marie Curie (Paris VI), Francia. Su interés en investigación se centra en la caracterización y efectos de inhibidores de la corrosión, así como en métodos de monitoreo de la corrosión.

Maura Casales-Díaz. Se desempeña como técnico académico en el Instituto de Ciencias Físicas de la Universidad Nacional Autónoma de México. Tiene la licenciatura y una maestría en ingeniería mecánica por la Universidad Autónoma del Estado de Morelos. Obtuvo el doctorado en ciencia de materiales por el Centro de Investigación en Materiales Avanzados, S.C. y trabaja en el campo de la corrosión y protección de materiales desde el 2000. Es investigadora nacional nivel I por el SNI.

José G. Chacón-Nava. Es investigador titular en el Centro de Investigación en Materiales Avanzados, S.C. Su experiencia de trabajo es en las áreas de corrosión en alta temperatura, ingeniería de materiales, análisis de fallas y monitoreo de la corrosión. Es ingeniero químico por el Instituto Tecnológico y de Estudios Superiores de Occidente, en Guadalajara, Jal. Tiene la maestría y el doctorado en ciencias de la corrosión e ingeniería por la Universidad de Manchester, Inglaterra, así como un postdoctorado en ingeniería de corrosión por la Benemérita Universidad Autónoma de Puebla. Sus intereses en investigación incluyen métodos de protección contra la corrosión i.e. inhibidores de corrosión, protección catódica y recubrimientos metalicos y no-metalicos. Es investigador nacional nivel II por el SNI.

Lorenzo Martínez-Gómez. Es investigador titular en el Instituto de Ciencias Físicas de la Universidad Nacional Autónoma de México. Tiene los grados de licenciatura, maestría y doctorado en física por la Facultad de Ciencias de la UNAM, así como un postdoctorado en ciencia de materiales por la Universidad de Stanford, USA. Sus principales líneas de investigación se relacionan con ciencia de materiales, metalurgia y el estudio de fenómenos de corrosión y métodos de protección. Ha dirigido varios proyectos sobre corrosión microbiana, corrosión asistida por esfuerzo y efecto de inhibidores de corrosión en ductos para transporte de hidrocarburos. Es investigador nacional nivel III por el SNI.

Alberto Martínez-Villafañe. Es investigador titular en el Centro de Investigación en Materiales Avanzados, S.C. Sus intereses en investigación incluyen el comportamiento de materiales en alta temperatura, recubrimientos metálicos y métodos de control de la corrosión. Tiene la licenciatura en físico-matemáticas y una maestría en ciencia de materiales por el Instituto Politécnico Nacional, así como el doctorado en ciencias de la corrosión e ingeniería por la Universidad de Manchester, Inglaterra. Es jefe del Departamento de Física de Materiales del CIMAV y consultor en corrosión del CONACYT. Es investigador nacional nivel III por el SNI.

José G. González-Rodríguez. Es investigador en el Centro de Investigaciones en Ingeniería y Ciencias Aplicadas de la Universidad Autónoma del Estado de Morelos. Es licenciado en física por la Universidad Autónoma de Nuevo León. Tiene una maestría y el doctorado en ciencias de la corrosión e ingeniería por la Universidad de Manchester, Inglaterra. Actualmente es jefe del grupo de ingeniería y ciencia de materiales del CIICAp. Es investigador nacional nivel II por el SNI. 